\title{
Speciation and hybridization in invasive fire ants
}

\author{
Pnina Cohen and Eyal Privman*
}

\begin{abstract}
Background: A major focus of evolutionary biology is the formation of reproductive barriers leading to divergence and ultimately, speciation. Often, it is not clear whether the separation of populations is complete or if there still is ongoing gene flow in the form of rare cases of admixture, known as isolation with migration. Here, we studied the speciation of two fire ant species, Solenopsis invicta and Solenopsis richteri, both native to South America, both inadvertently introduced to North America in the early twentieth century. While the two species are known to admix in the introduced range, in the native range no hybrids were found.

Results: We conducted a population genomic survey of native and introduced populations of the two species using reduced representation genomic sequencing of 337 samples. Using maximum likelihood analysis over native range samples, we found no evidence of any gene flow between the species since they diverged. We estimated their time of divergence to $190,000(100,000-350,000)$ generations ago. Modelling the demographic history of native and introduced S. invicta populations, we evaluated their divergence times and historic and contemporary population sizes, including the original founder population in North America, which was estimated at 26 (10-93) unrelated singly-mated queens.

Conclusions: We provide evidence for complete genetic isolation maintained between two invasive species in their native range, based, for the first time, on large scale genomic data analysis. The results lay the foundations for further studies into different stages in the formation of genetic barriers in dynamic, invasive populations.
\end{abstract}

\section{Background}

The separation of populations and their eventual speciation is a fundamental process and a driving force in evolution. However, a newly formed reproductive barrier is often incomplete. Hybridization and introgression between incipient species, or gene flow from one species' gene pool into another by backcrossing, may still occur. Many cases of species hybridization, resulting from accidental introduction of foreign species into new environments, have been identified and documented [1-3]. Often the outcome of human activities, these systems provide an opportunity to dissect the mechanisms underlying the formation of reproductive barriers. Exploring the differences between populations and their environments in native and introduced ranges may reveal the factors responsible for genetic isolation, which would eventually result in irreversible speciation.

\footnotetext{
* Correspondence: eprivman@univ.haifa.ac.il

Department of Evolution and Environmental Biology, Institute of Evolution, University of Haifa, Haifa, Israel
}

Here, we describe one such case study involving two invasive fire ant species. These species, thought to maintain genetic isolation in their native range [4-6], freely hybridize in their newly introduced range $[7,8]$. Unlike other incidents of introduction resulting in admixture, the two species are parapatric in both the native and the introduced ranges. Furthermore, the fact that the two ranges are located thousands of kilometers apart, allows for clear distinction between the native and introduced populations under study. This makes it a unique system which can help to provide insights into the evolution of genetic isolation and speciation.

The red fire ant, Solenopsis invicta, and the black fire ant, S. richteri, are closely related species, native to South America. Both species were inadvertently introduced to North America in the early twentieth century [9-11], with S. invicta subsequently migrating to other countries worldwide $[12,13]$. Since they were first detected in the USA, the fire ant species have been closely monitored and their spread across the southeast is well 
documented. Their life cycle, behavior, genetic makeup, population structure and invasion history are the subject of many publications, making these species, in particular S. invicta, amongst the most studied invasive species, and an excellent subject for demographic history analysis.

S. invicta and S. richteri' display social polymorphism, with two distinct social colony structures - the monogyne (single queen) and the polygyne (multiple queens) forms. The social polymorphism is a Mendelian trait determined by a supergene of 13 megabases, and is marked by the gene $g p-9$ with monogyne queens always having $g p-9^{B B}$ genotypes and polygyne queens always having $g p-9^{B b}$ genotypes [14-16]. The social structure of a colony greatly affects its size, longevity, and dispersion patterns $[17,18]$.

Population genetics analysis of microsatellite and mitochondrial genotypes of thousands of S. invicta colonies around the world were used to trace the place of origin of the introduced S. invicta populations to the Formosa region in northern Argentina [11, 13]. It was also established that all subsequent introductions of $S$. invicta across the globe originated in the introduced USA population. The population of origin of the introduced $S$. richteri is still unknown.

The number of S. invicta queens that were initially introduced to North America was estimated through the screening of haplotypes in mtDNA sequences, as well as genotypes of allozymes, microsatellites, and the complementary sex determination (CSD) locus in individuals sampled throughout the introduced range [19]. It was determined that to account for all of the allele variants, the original group that was introduced to USA consisted of 9-30 unrelated mated queens.

In the introduced range, admixture between S. invicta and $S$. richteri is prevalent $[7,8]$. A hybrid zone was identified ranging from Georgia in the east through Alabama to central Mississippi [18, 20, 21]. This is not the case in the native range of South America, where admixture was found to be a rare occurrence [4], or not evident at all [4-6]. While extensive sampling of native colonies was conducted, no more than 26 nuclear genetic markers were used in testing for admixture between the species, which limits the power of the analysis to detect gene flow.

We used large scale population genomic data to study the demographic history of S. invicta and S. richteri. Our data include samples from both species in both native and introduced ranges. The hybrid zone was not included in the sampling because the admixture in the introduced range has been well established in previous studies $[7,8]$. These genomic data allow for an explicit maximum likelihood test for historic gene flow between the $S$. invicta and S. richteri in their native range to determine if these species had indeed maintained genetic isolation since their separation. We also provide estimates for speciation times, population divergence times and historic and contemporary effective population sizes, including the founding S. invicta population in North America. This is the first demographic history study of these species that uses thousands of genomic markers, which allow population genetic model inference at high accuracy and provide the necessary statistical power to test for gene flow.

\section{Results}

We inferred the population structure and demographic history using RAD sequencing of population samples of $S$. invicta and S. richteri from nine localities in their native range in Argentina and their introduced range in the USA (Fig. 1; Additional file 1: Tables S1 and S2). Altogether, this dataset consists of 962,896,602 sequenced reads, 96 nucleotides long each, from 337 samples. After stringent quality filtering, the genotypes of between 6389 and 285,847 SNPs, representing between 161 and 337 individuals (depending on filtering parameters and analysis type) and a total of 16,648 aligned RADseqs were used in the different analyses (see Methods).

\section{Population structure}

STRUCTURE $[22,23]$ is a Bayesian clustering method that assigns individuals to ancestry clusters. STRUCTURE analysis resulted in the identification of five distinct population clusters (Fig. 2). No further substructure was found for $K>5$ (Additional file 1: Figure S1). In 76 of 100 STRUCTURE runs with $K=5$, the four introduced populations were clustered into two separate clusters, one for each species. Two individuals of the introduced S. richteri population of Benton, Tennessee, had inferred ancestry belonging to both clusters, indicating possible hybrids. The two native $S$. richteri populations were assigned to a single cluster, while two of the native S. invicta populations (Alejandra and El Recreo) were assigned to a fourth cluster. Individuals of the third native $S$. invicta population, sampled in Herradura, were partially assigned to the cluster of the introduced $S$. invicta populations and partially to a separate, fifth cluster.

The allocation of populations into clusters suggests that the polymorphism in the introduced $S$. invicta is a subset of the genetic variants found in the native Herradura samples, making the latter a possible population of origin of the former. This result is in line with the previous studies that identified the Formosa region, which includes Herradura, as the likely source of the introduced $S$. invicta $[11,13]$. Unlike the pattern observed in the Herradura population, neither of the sampled native $S$. richteri populations share a cluster with the introduced 

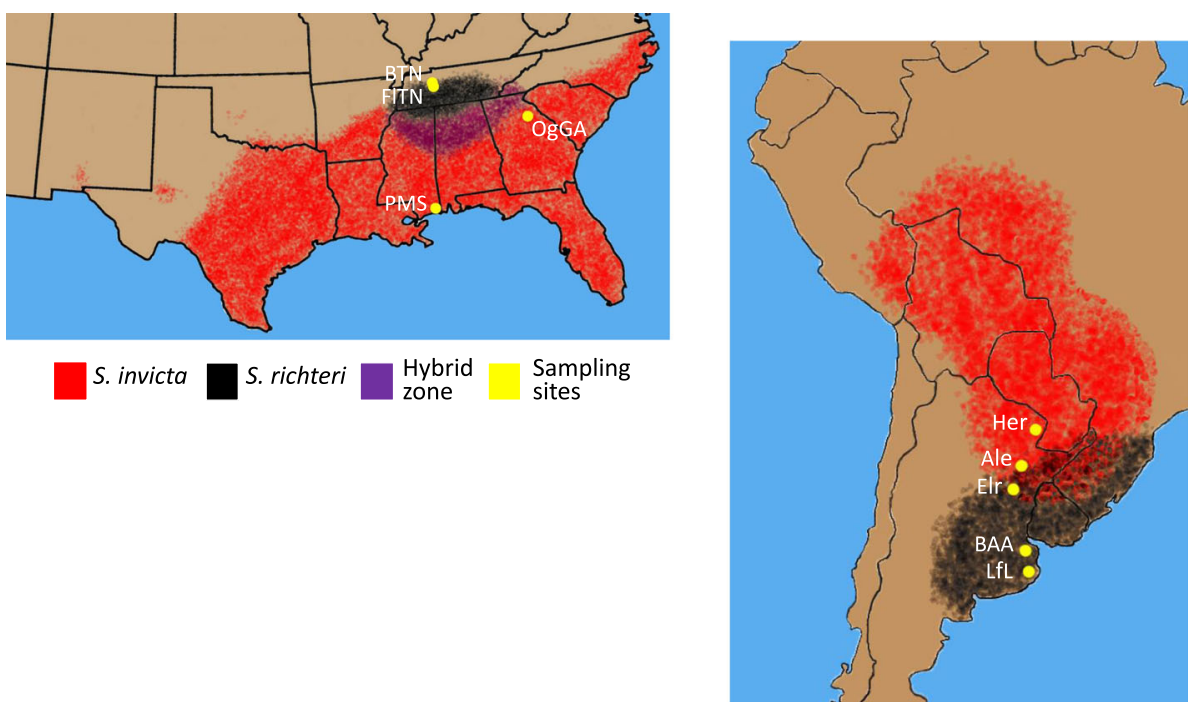

Fig. 1 Distribution map of S. invicta and S. richteri across North and South America with marked sampling sites. S. invicta was sampled in three sites in its native range (Her, Ale, Elr) and in two sites in the introduced range (OgGA, PMS). S. richteri was sampled in two sites in its native range $(B A A, L F L)$ and in two sites in the introduced range (BTN, FINT). The area marked in purple shows the hybridization zone between $S$. invicta and S. richteri. Distribution is based on [13, 60-63] and https://www.aphis.usda.gov/plant_health/plant_pest_info/fireants/downloads/fireant.pdf. Sampled populations are: Herr - Herradura, Elr - El Recreo, Ale - Alejandra, PMS - Pascagoula, Mississippi, OgGA - Oglethorpe Co., Georgia, BAA - Buenos Aires, LfL - Las Flores, BTN - Benton Co., Tennessee FITN - Flatwood, Tennessee

populations, indicating that these are not the source population of the introduced S. richteri.

We calculated the genetic distances between each pair of populations, using the genetic differentiation measure of $F_{S T}$. The results can be found in Additional file 1: Table S3.

\section{Reproductive isolation in the native range}

We tested for historic and contemporary gene flow between the native $S$. invicta and S. richteri populations by comparing between two competing demographic history models. To this end, we used a full maximum likelihood analysis of coalescent models [24, 25] as implemented in the $3 s$ software [26]. Based on the phylogenetic inference, the maximum likelihood scores of two possible demographic history models are compared. A null model that does not allow genetic flow between the two closely related species and an alternative model that does.

This analysis concluded with an insignificant difference between the likelihood scores of the two scenarios $(\Delta \ln L=0.06 ; p>0.9)$. Consequently, the null model could not be rejected, indicating that there was no evidence of gene flow between the native $S$. invicta and $S$. richteri populations since their divergence from a common ancestral population.

\section{Speciation times and population sizes}

$3 s$ provided maximum likelihood estimates for the null model parameters, including effective population sizes and the time since the speciation of S. invicta and $S$. richteri. Since we used the sequence of an individual of a

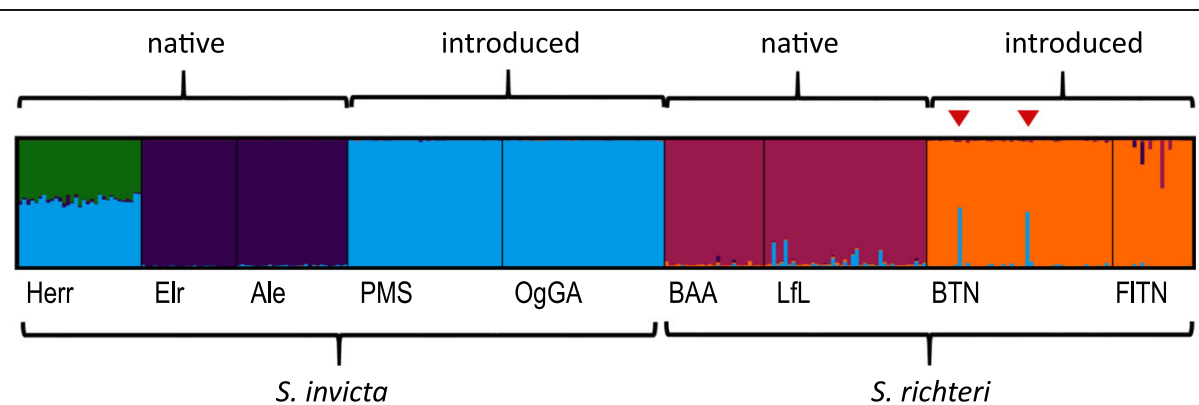

Fig. 2 Structure analysis of the studied populations. Summary of 76 of 100 runs of STRUCTURE with $K=5$. Each individual is shown as a vertical bar, colored in accordance to its inferred clustering. Individuals of eight of the nine populations are clustered according to species and range, with the exception of two individuals marked in red triangles. These are mapped to both introduced S. invicta and S. richteri clusters, probably due to hybrid ancestry. Individuals of Herradura population are mapped to a fifth cluster, and to a cluster shared with the introduced S. invicta ants 
different Solenopsis species, Solenopsis fugax, as an out-group, the model provided an estimate to the speciation time of this species from the lineage leading to $S$. invicta and S. richteri. Estimates were also provided to the effective size of the populations of S. richteri and $S$. invicta, the effective size of the ancestral population of S. invicta and S. richteri and that of the ancestral population of all three Solenopsis species (Table 1). Using the mutation rate of $3.4^{*} 10^{-9} \mathrm{bp} /$ generation $(95 \%$ confidence interval of $2.2^{*} 10^{-9}-4.9^{*} 10^{-9}$ ) that was estimated for the honey bee $[28,29]$, we calculated that the speciation of S. invicta and S. richteri happened $1.9^{*} 10^{5}\left(1^{*} 10^{5}\right.$ $3.5^{*} 10^{5}$ ) generations ago and the speciation of $S$. fugax from the lineage leading to $S$. invicta and S. richteri took place $2.5^{*} 10^{6}\left(1.6^{*} 10^{6}-4 * 10^{6}\right)$ generations ago. See Fig. 3 for all estimated parameters.

The estimates for the effective population sizes were similar between present-day populations of $S$. invicta and $S$. richteri, while the ancestral population of these two populations was found to be slightly smaller. The effective size of the ancestral population of the three Solenopsis species was found to be larger than the other populations by an order of magnitude, indicating a very diverse ancestral population. See Fig. 3 for the exact numbers.

\section{Founder population of introduced S. invicta}

We estimated the demographic and temporal parameters that define the history of $S$. invicta populations using Approximate Bayesian Computation (ABC) [30], as implemented in $D I Y A B C$ v2.0.4 [31, 32]. Unfortunately, it was not possible to fit a similar model to the $S$. richteri populations (poor goodness of fit). This may be because we did not sample the source population of the S. richteri introduction.

An ABC analysis is often used to compare between multiple competing historic demographic models that differ in their scenarios. As many details in the history of S. invicta are already established, an alternative scenario is unnecessary. Rather, we concentrated on estimating the model parameters of population sizes and time of demographic events as depicted in Fig. 4.

With a maximum posterior of 39 (95\% credible interval of 14-139), the effective size of the founding population is quite low. The length of the bottleneck period of the introduced S. invicta $\left(T_{B}\right)$ is minimal and stands at 2 generations for the maximum posterior estimate, with
95\% credible interval ranging between 0 and 16 generations. Combined with the small size of the founder population, this suggests a rapid increase in population size immediately following the introduction. It is in line with historic records in which the presence of the newly introduced fire ants was initially reported as a mere curiosity and quickly changed into a growing concern and even panic, as ant numbers exploded [18].

The effective population size of the Herradura population $\left(N_{N I}\right)$ is approximately two orders of magnitude larger than the second native population cluster $\left(N_{N 2}\right)$ estimated at $1.2^{*} 10^{7}$ and $2.5^{*} 10^{5}$, respectively (95\% credibility interval of $3.3^{*} 10^{6}-7.8^{*} 10^{7}$ and $8.2^{*} 10^{4}-2^{*} 10^{6}$, respectively).

In Fig. 5, we plotted the full posterior distributions of all model parameters. The numeric values of these distributions, including their 95\% quantiles, are summarized in Additional file 1: Table S4. For parameters $N_{I}, T_{I}$ and $T_{D}$, the posterior density distributions are very similar to their prior density distributions, indicating that the analysis could not extract information from the given data. The first two parameters are directly linked to the recent introduction to the USA, and the rapid increase in population size that followed. The sudden shift in dynamics from the originally stabilized population in the native range may have resulted in poor representation of the introduced populations by their summary statistics. Nevertheless, a 'goodness of fit' analysis indicated that the observed genetic data are well explained by the model and its parameter posterior distributions (Additional file 1: Figure S4).

\section{Discussion}

We explicitly tested two competing demographic history models and found that the reproductive barrier between two closely related species of fire ants was sustained for about 200,000 generations, and is still preserved in their native range of South America. The STRUCTURE analysis of 171 native samples seems to indicate low levels of introgression of S. invicta ancestry into the native $S$. richteri population in Las Flores population, but not in the Buenos Aires population. If hybrids are indeed formed on rare occasions, it may be that such incidents do not lead to detectable gene flow between the species. Alternatively, this may be noise in the STRUCTURE analysis. The latter explanation seems more likely because

Table 13 s parameter estimates

\begin{tabular}{lllll}
\hline & $\begin{array}{l}\text { Ancestral population } \\
\text { to S. invicta and richteri }\end{array}$ & $\begin{array}{l}\text { Ancestral population to } \\
\text { S. invicta, richteri and fugax }\end{array}$ & S. richteri & S. invicta \\
\hline Temporal parameters & $0.000646(0.00052-0.00077)$ & $0.008369(0.00789-0.00884)$ & & \\
Demographic parameters & $0.002293(0.00211-0.00247)$ & $0.026874(0.02591-0.02783)$ & $0.003596(0.00309-0.00409)$ & $0.004062(0.00343-0.00469)$ \\
\hline
\end{tabular}

3 s estimates of the null model parameters in the highest scoring run 


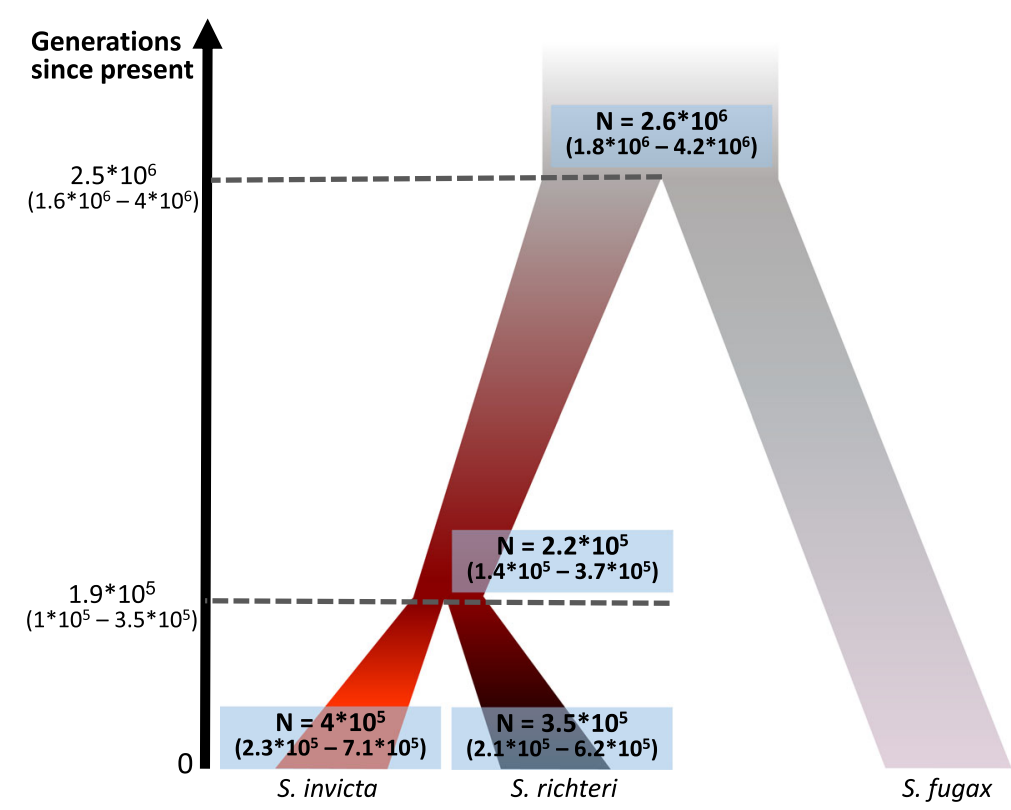

Fig. 3 Population effective sizes and generations number since the two speciation events. The values were calculated using the mutation rate of $3.4^{*} 10^{-9}$ with the $95 \%$ confidence level range of $2.2^{*} 10^{-9}-4.9^{*} 10^{-9}$ over $3 S^{\prime}$ s estimates (Table 1). Marked in blue are the population effective sizes and to the left is the time line in generations number

Las Flores is more distant from the S. invicta range than Buenos Aires.

A previous study conducted by Ross and Trager (1990) indicated that genetic isolation may be incomplete in the native range [4]. By analyzing six informative allozyme loci (out of 26 that were genotyped) in 100 samples of S. invicta and 57 samples of S. richteri, three samples were identified as possible hybrids. The results were inconclusive, but occasional admixture between $S$. invicta and S. richteri could not be dismissed. Neither could it be determined if the hybrid specimens, assuming it is what they were, are the product of one or many generations of admixture, and so the reproductive potential of native invicta-richteri hybrids was still unclear. Other studies that used less extensive sampling and more diverse, but still limited, genetic markers of mtDNA sequences and allozyme loci, could identify no signs of admixture $[5,6]$. Our conclusion of genetic

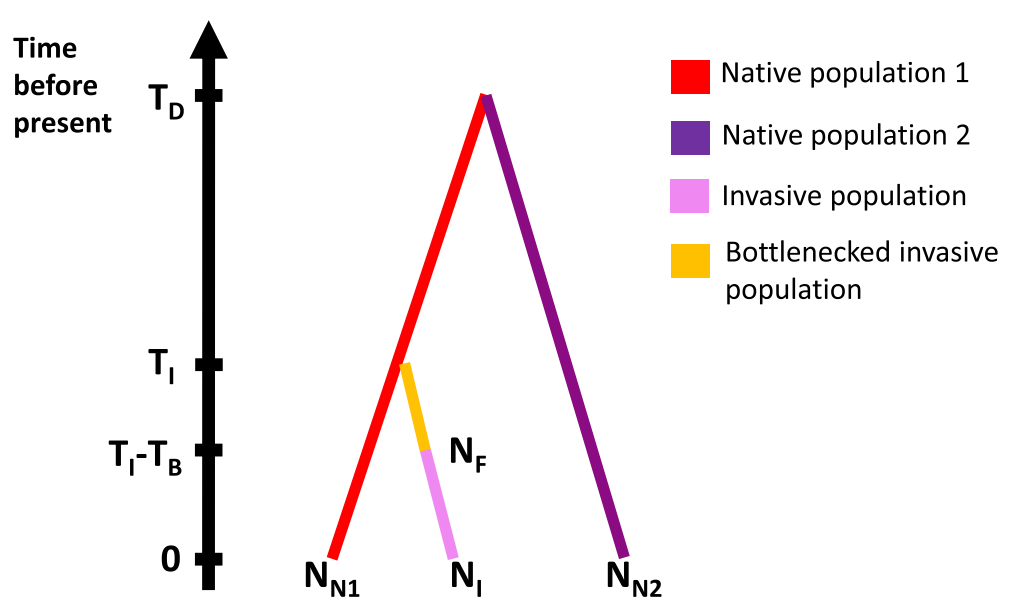

Fig. 4 Model of S. invicta demographic history. The model describes the population history starting with the divergence within the native populations and followed by the introduction to North America, which originated from the Herradura population. Populations: $N_{N 1}-$ native population of Herradura; $N_{N 2}$ - native population of Alejandra and El Recreo; $N_{F}$ - bottlenecked founder population in the USA; $N_{l}$ - contemporary population in the USA. Times: 0 - populations sampling; $T_{1}$ - introduction; $T_{B}$ - length of bottleneck for the introduced population; $T_{D}$ - divergence of the Herradura population from the Alejandra and El Recreo populations 


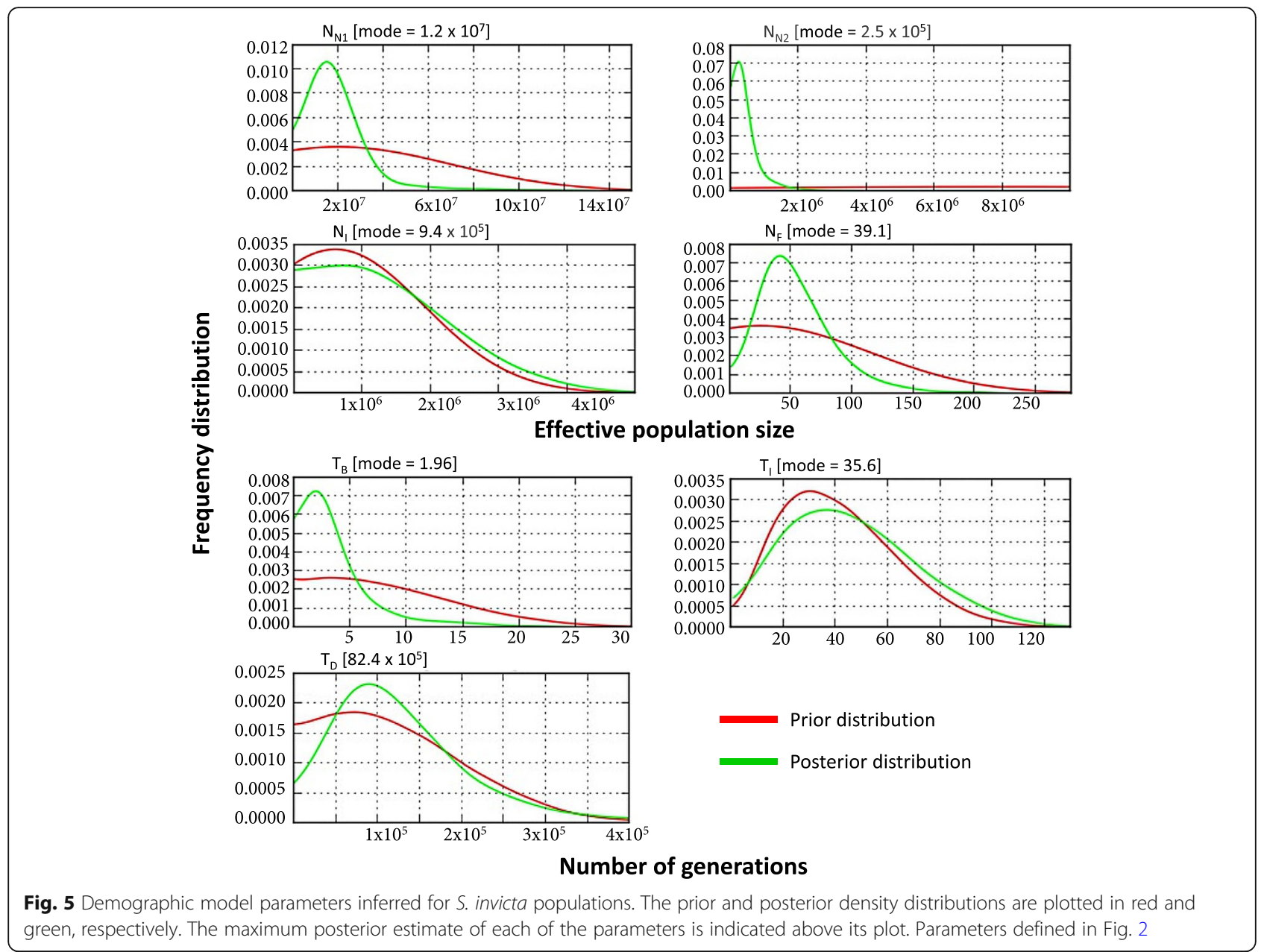

isolation between these species since they diverged is more powerful than the observation that admixture is not taking place at present time, and is consistent with a strong reproductive barrier. It makes the extensive admixture, observed in North America, even more intriguing. This suggests that the two diverging lineages of $S$. invicta and $S$. richteri were well on their way to complete speciation in South America, but have not reached the point of no return. Their introduction to the USA has resulted in the breakdown of barriers and reversal of this process.

Reproductive isolation between related species is maintained by endogenous (genetic) and/or exogenous (environmental) factors [33]. One explanation for the difference between the admixture patterns in the native and introduced ranges could be that the native populations that were the sources of the introductions are genetically compatible between the species but geographically separated, while the populations whose ranges overlap in South America, are incompatible. This explanation was previously proposed by Ross \& Trager (1990) [4]. Since Formosa is located outside of the overlap region, this may well be the case. Another possibility is that the introduced populations have been released from certain exogenous factors, biotic or abiotic, that prevent admixture in the native range. Biotic factors may include parasites that affect mating and reproduction. One such factor is Wolbachia - an endosymbiotic bacterium that infects high proportion of insects [34], and manipulates their reproductive systems in ways such as the induction of cytoplasmic incompatibilities between infected and uninfected individuals. Such incompatibilities were found to hinder the admixture between two species of Drosophila [35]. As Wolbachia was detected in native populations of $S$. invicta and S. richteri, but not in the introduced populations [36] it is a strong option that was already pointed out before [6]. Another possibility is changes in the ants' diet following their introduction to a new environment. Different foods might affect the chemical blend of pheromones the ants produce. In ants and in other insects, social interaction, which includes the identification of a potential sexual mate, relies heavily on chemical cues [37]. In fact, pheromonal differentiation is suspected to be the cause of reproductive isolation between many closely related insect species [38-40]. 
Moreover, diet was shown to affect the pheromonal profile and sexual desirability in fruit flies [41, 42].

We gave estimates of the number of generations since the two speciation events, which can be converted to years by multiplying them with the average generation time. The social polymorphism of S. invicta and S. richteri combined with overlapping generations and multiple nuptial flights throughout the year, make the length of a generation highly variable and difficult to pinpoint. Ross and Shoemaker (2008) have given an estimate of three to six years, based on the relative number of alates produced in different developmental stages of a colony [19]. They lean towards the higher end of this range, but maintain that a shorter generation time is more probable for the time following introduction, when their numbers were growing exponentially. Assuming an average generation time of six years, the speciation time between $S$. invicta and S. richteri can be estimated to be $1.1(0.6-2.1)$ million years ago (MYA) and the speciation time of S. fugax and S. invicta and $S$. richteri to 15 (9.6-24) MYA. The high end of this estimate is just under the 25 million years (95\% confidence interval of 18-32) inferred for the divergence of these lineages, based on Bayesian phylogenetic analysis and divergence dating using 27 fossil calibration points [43]. It should be noted that a phylogeny-based estimate is for the genetic distance between the two representative individuals whose sequences were analyzed, while the coalescent-based-estimate by $3 s$, is for the separation of the species (i.e. the formation of genetic isolation). By definition, the divergence between the representative individuals must predate the formation of reproductive barriers that led to speciation.

There is one parameter shared by the two different coalescent-based methods of $D I Y A B C$ and $3 s$, which is the native $S$. invicta populations cluster, that of Alejandra and El Recreo. Both methods gave similar estimates for it $\left(2.5^{*} 10^{5}\right.$ and $4^{*} 10^{5}$ respectively). It is approximately two orders of magnitude smaller than $D I Y A B C$ 's estimate of $1.2 * 10^{7}$ given for the other native population cluster, the Herradura population. This suggests a much larger and more diverse population around Herradura in comparison to the southern population sampled at El Recreo and Alejandra. Of those three locations, Herradura is closest to Corrientes, which was highlighted as the most genetically diverse among the populations that were sampled across South America [44].

The effective population size of the $S$. invicta USA founder population $\left(N_{F}\right)$ was estimated at 39 . While for diploid species the effective population size is $\mathrm{Ne}$ $=\frac{4 \mathrm{NfNm}}{\mathrm{Nf}+\mathrm{Nm}}\left(N_{f}\right.$ and $N_{m}$ are the number of breeding females and males, respectively), for haplodiploid species, the reduced number of chromosomal copies in males results in $\mathrm{Ne}=\frac{4 \mathrm{NNNm}}{2 \mathrm{Nf}+4 \mathrm{Nm}}[45,46]$. This translates to a ratio of $N_{e}$
$=1.5 N_{f}$ for species in which the queen mates with a single male, as is the case with S. invicta [47]. Therefore, the founder population size maximum posterior estimate of 39 translates to 26 unrelated, singly-mated queens, which fits within the higher end of the 9-30 estimate range made by Ross and Shoemaker (2008). If we consider our entire $95 \%$ credible interval, then the number of founding S. invicta queens is between 10 and 93.

\section{Conclusion}

We described a unique study system in which the reproductive barrier between two closely related species is maintained for approximately 200,000 generations in their native range and breached in an introduced range. This setting allows sampling from clearly separate pure populations in the native range and admixing populations in the introduced range. Therefore, this is a powerful system for the study of the molecular basis of reproductive isolation, which is key to the process of speciation.

\section{Methods}

\section{Population sampling}

Samples were taken from nine ant populations: three $S$. invicta and two S. richteri populations in the native range in Northeastern Argentina; two S. invicta and two S. richteri populations in the introduced range in Southeastern USA. The specimens were identified by morphological characteristics by James Pitts as described before [48]. For each population, 23-51 diploid females were sampled, each from a different colony, totaling 337 individuals. See Fig. 1 and Additional file 1: Tables S1 and S2 for details.

\section{Sequencing and processing}

Seven restriction-site associated DNA (RAD) libraries were constructed from the sampled populations, with 31-68 individuals in each library. DNA was extracted from the samples and RAD libraries were constructed based on the protocols of Baird and colleagues [49] and Etter and colleagues [50], as described by Privman et al. (2018) [27]. Briefly, DNA was digested with PstI-HF enzyme (New England Biolabs) and ligated to one of 96 barcoded P1 adapters with 5 bp unique barcodes. The samples were multiplexed per lane of $100 \mathrm{bp}$ single-end sequencing on an Illumina HiSeq 2000 or 4000 sequencer. A total of $962,896,602$ reads, or RADseq, were sequenced, an average of 2,857,259 reads per sample, with the least number of reads for a sample being 197,598 and the most - 6,848,114. Library 3 averaged at the lowest number of reads of $1,628,368$ reads per sample. Library 7 averaged at the highest number of reads of 3,335,288 reads per 
sample. See Additional file 1: Table S1 and S2 for details about samples, reads and sequencing depth.

The raw reads were initially processed using the Stacks pipeline $[51,52]$ and low quality reads were discarded. These were defined as reads in which the phred score drops below 10 (1:10 chance for a sequencing error), averaged over a sliding window of 14 bases. We mapped the reads to 44,102 distinct positions in a S. invicta reference genome (version $\mathrm{Si}$ gnH; NCBI accession AEAEQ02000000; [27]) using Bowtie2 [53]. For each of the mappings, a maximum of two bases mismatch was allowed for its best hit. To maintain the uniqueness of the mapping, we removed alignments with the second best hit containing less than five bases mismatches. Additionally, we controlled for and filtered out reads mapped to what are suspected collapsed repetitive sequences in the reference genome assembly; these were identified based on excessive coverage and heterozygous genotype calls in whole-genome sequencing of 40 haploid S. invicta males sampled from Herradura and Alejandra [27].

We analyzed the mapped sequences for single nucleotide polymorphic sites (SNPs) using the Stacks pipeline and created a catalog containing 285,847 SNPs for the combined seven RAD libraries, with measured coverage of X16 on average. These SNPs were used to calculate the $F_{S T}$ distances between the populations.

\section{Population structure}

We filtered the SNPs catalog further before its use in the population structure analyses. For each locus, we required genotype calls in at least $80 \%$ of the samples in each population (i.e. less than $20 \%$ missing data); a minimum of three reads made for a genotype call. Additionally, we required a minimal minor allele frequency (MAF) of $1 \%$. This stringent filtering resulted in a collection of 16,759 high confidence SNPs. Finally, we removed samples with more than $30 \%$ missing data (i.e. loci without genotype calls), leaving 300 samples in the analysis.

We ran STRUCTURE over $K$ (number of expected clusters) values of 2-9, 100 times for each $K$, with 100 different sets of 1000 SNPs randomly chosen out of the high confidence SNPs collection, a total of 800 separate runs. To avoid linked sites from affecting the analysis, we required a distance of at least $5000 \mathrm{bp}$ between the selected sites. Multiple runs over various data subsets contributed to the robustness of the clustering inference and allowed us to use more SNPs than would be possible in a single run. We ran STRUCTURE for 1,100,000 MCMC repetitions and discarded the first 100,000 (burn-in period). All the other parameters were kept at default values. The results were analyzed using CLUMPAK [54]. The STRUCTURE analysis was repeated with all the SNPs in the Social chromosome removed, leaving 15,539 SNPs in the analysis. The results were largely unchanged, and they can be found in Additional file 1: Figure S2. We used the Evanno test [55] to identify the number of clusters $\mathrm{K}$, which gave $K=4$ (Additional file 1: Figure S3). However, we decided to present the results for $\mathrm{K}=5$ where the native and introduced $S$. richteri separated to two distinct clusters.

\section{Populations demographic history \\ Full maximum likelihood test for gene flow using $3 \mathrm{~s}$}

We tested the hypothesis that S. invicta and S. richteri have been maintaining complete genetic isolation in South America since their divergence. The alternative to this scenario is that these incipient species have had some form of gene flow between them, including isolated successful mating events (isolation with migration). We used $3 s$ program, which is a coalescent-based maximum likelihood inference tool. The program analyses aligned sequences of three species, two that are closely related and a third which is used as an out-group, for the genealogical process of coalescence and migration. Based on this the maximum likelihood scores of two possible demographic history models are compared, a null model that does not allow genetic flow between the two closely related species and an alternative model that does.

In addition, $3 s$ provides estimates for the times of the two speciation events and for historic and contemporary population effective sizes. Speciation times are inferred from the model parameter $\tau$ - the average number of substitutions per site since speciation. Effective population sizes are represented by the $\theta$ parameter. In diploid species, $\theta=4 \mu \mathrm{N}_{\mathrm{e}}$, where $\mu$ is the mutation rate and $N_{e}$ is the effective population size. For a haplodiploid species, $\theta=3 \mu N_{e}$ because of the ploidy ratio between males and females.

Using $3 s$, we investigated the demographic history of the closely related $S$. invicta and S. richteri species with the thief ant $S$. fugax as an out-group (genome assembly version Sf_gnA; NCBI accession QKQZ00000000; [27]). To give meaning to the chosen model parameter estimates of speciation times and effective population sizes, we used the mutation rate of another hymenopteran, the honeybee, which was estimated at $3.4 * 10^{-9}$ (95\% confidence interval of $2.2^{*} 10^{-9}-4.9^{*} 10^{-9}$ ) mutations per site per generation. This mutation rate was calculated using direct measurement of two generations in three colonies and is similar to the mutation rate inferred for other insects $[56,57]$.

Genetic data set preparation The program requires only a small number of individuals with a large number of loci to represent each species. We arbitrarily chose two $S$. richteri individuals from the populations of Las Flores and Buenos Aires $\left(R_{1}\right.$ and $R_{2}$ respectively) and 
two $S$. invicta individuals from the populations of Alejandra and El Recreo ( $\mathrm{I}_{1}$ and $\mathrm{I}_{2}$ respectively). All four chosen individuals were of the $g p-9^{B B}$ genotype of the social chromosome. Using Bowtie2, we aligned their RAD reads to the reference genome of $S$. invicta and to a fully sequenced S. fugax individual, allowing no gaps in the alignments. We retained only RADseq reads with no more than 4 mismatches compared to the S. invicta reference genome and RADseq reads with no more than 10 mismatches compared to the $S$. fugax genome. The mismatches cutoffs are based on the average of $95 \%$ sequence identity between S. invicta and S. fugax [27]. We also required unique mapping and only allowed alignments in which the second best hit has at least twice the number of mismatches as the best hit.

We assembled for each locus an alignment of three sequences composed of the sequences of the outgroup of S. fugax and one of following pairs of sequences at random: $I_{1}$ and $I_{2}, R_{1}$ and $R_{2}$ or $R_{1}$ and $I_{2}$. As the algorithm assumes no linkage disequilibrium $(L D)$ between sites, we only allowed loci that were at least 2000 nucleotides apart. This produced 15,040 triplets of 96 bases long three-ways alignments, to be used as input for $3 s$ software.

Execution We ran $3 s$ and calculated the maximum likelihood values for the two models using the Gaussian quadrature number of points $=16$. To examine the robustness of the estimates we ran each of the models three times, with different seed values at each run. We used the likelihood scores and the parameter values that were obtained for the highest scoring run of each of the models and compared them in a Likelihood Ratio Test (LRT).

\section{Demographic history of populations of S. invicta}

Using approximate Bayesian computation (ABC), we estimated divergence times and population sizes of the three S. invicta population clusters identified by STRUCTURE, for the demographic history model depicted in Fig. 4. Instead of full Bayesian calculations of a likelihood function, $D I Y A B C$ uses an approximate approach: it runs a series of coalescent simulations over a demographic history model, which includes an historic scenario and parameter prior distributions. The program estimates the posterior distributions for the model parameters based on how well the simulated data fit the observed genetic data (represented by their summary statistics).

Our STRUCTURE analysis found the two native $S$. invicta populations of Alejandra and El Recreo indistinguishable in term of their genetic polymorphism and so they were assigned to one population, while Herradura's population was clustered separately. The introduced populations were also clustered together in one population. We formulated the demographic history model used for the ABC analysis with these three distinct population clusters.

Scenario construction In the historic scenario, depicted in Fig. 4, the two native populations, $N_{N 1}$ and $N_{N 2}$, diverged from each other $T_{D}$ generations ago, prior to the introduction to the USA, which took place $T_{I}$ generations ago. As the introduced populations in the USA were found to originate from the region of Formosa (which includes Herradura), in our scenario the founder population, $N_{F}$ is directly derived from population $N_{N 1}$, and their divergence time coincides with the time of introduction. The scenario also includes a population bottleneck effect after the introduction, which lasted for $T_{B}$ generations and concluded as the size of the population reached its contemporary size, $N_{I}$.

Parameterization Coalescent model parameters are either demographic, reflecting population effective sizes, or temporal, indicating the number of generations that had passed between events. We defined parameter priors of normal distributions with averages based on known life history of the fire ants. Prior distribution widths were set widely to allow $D I Y A B C$ sizeable sampling range, encompassing all reasonable values of these parameters.

The introduction time of $S$. invicta predates sampling time by at least 80 years. Generation time for the fire ants, especially at the time they were dispersing throughout the newly introduced range, is hard to estimate. We therefore defined a prior of introduction time that ranges between 0 to 180 generations. Based on studies indicating that the original number of introduced $S$. invicta was small, we defined a prior for the effective population size that ranges between 1 and 500 individuals. The ants' quick expansion in North America suggests a minimal time span of the population bottleneck. This allowed us to narrow the prior distribution of the bottleneck time between 0 and 40 generations. Not much is known about the effective sizes of the native and introduced populations or of the time of divergence between the native populations, and these parameter priors were kept at a wide distribution, which included many orders of magnitudes.

Choice of summary statistics An ABC analysis depends on population summary statistics to reduce the high-dimensional genetic information and to evaluate the simulation results. A varied choice of summary statistics is therefore crucial. However, using too many would increase the dimensionality of the analysis, making the available observed data points too sparse in comparison [58]; a problem known as "the curse of dimensionality". 
The native $N_{N 1}$ and $N_{N 2}$ populations are presumed stable and well represented by their summary statistics. We therefore used all of the within-population summary statistics offered by $D I Y A B C$ : the proportion of monomorphic alleles, mean and variance of gene diversity of polymorphic loci [59] and mean gene diversity across all loci. For the population $N_{I}$, a newly established population, we only used the summary statistics of the proportion of monomorphic alleles and mean gene diversity across all loci.

We included between-populations summary statistics for pairs of populations that directly diverged from each other $\left(N_{N 1} \& N_{N 2}\right.$ and $\left.N_{N 1} \& N_{I}\right)$ : the mean $F_{S T}$ distance, the proportion of null $F_{S T}$ distances [60], and mean and variance of non-null $F_{S T}$ distances.

Genetic data set preparation We subdivided the SNPs catalog to create a dataset to include only the $S$. invicta samples. The SNPs were filtered as described before, with the following modifications: we required that each locus has genotype calls in at least $90 \%$ of the samples of each population, a minimal MAF of $0.5 \%$, and at least 5000 bp between SNPs. The reduced MAF threshold is meant to allow the $D I Y A B C$ analysis to make use of the valuable information in the low frequency alleles. This resulted in 161 samples and 6389 loci that were used in the analysis.

Simulations and analyses Running $D I Y A B C$ coalescent simulations, we created 892,000 simulated data sets. Based on the $1 \%$ of the simulations that produced summary statistics closest to the observed data, the parameter posterior distributions were estimated and adjusted using a weighted linear regression in which the summary statistics were the independent variables. To measure the goodness of fit of the model [61], we randomly selected $10 \%$ of the adjusted simulated data sets, and compared them to the observed data in a PCA analysis of the summary statistics.

\section{Additional file}

Additional file 1: Table S1. Populations sampling times, locations, numbers and social form genotypes. Also detailed is the average coverage per ant for each of the population after the completion of initial filtering to the point of unique mapping to the reference genome. Table S2. RAD libraries composite and raw reads number. Figure S1. STRUCTURE'S major run results for $\mathrm{K}=2-9$. Figure S2. STRUCTURE'S major run results for $\mathrm{K}=2-9$ with SNPs linked to chr16 (social chromosome) removed, leaving 15,539 SNPs in the analysis. Populations as before. Figure S3. Evanno analysis for best K. Table S3. - $F_{S T}$ between populations. Table S4. Posterior distributions of S. invicta demographic history model parameters. Figure S4. Goodness of fit analysis result for $\mathrm{S}$. invicta demographic history model. (DOCX $446 \mathrm{~kb}$ )

\section{Abbreviations}

ABC: Approximate Bayesian computation; CSD: Sex determination locus; LD: Linkage disequilibrium; MAF: Minor allele frequency; MYA: Million years ago; RAD: Restricted site associated DNA; RADseq: RAD sequence; SNP: Single nucleotide polymorphism

\section{Acknowledgments}

We are especially grateful for the generous contributions of DeWayne Shoemaker with difficult to obtain samples, genomic sequencing, and advice on fire ant demography, population biology, ecology, and evolution throughout the project. We also thank Ziheng Yang, Alan Templeton, Eran Halperin, and Abraham Korol for very helpful advice on the population genomic analyses.

\section{Funding}

This work was supported by a US-Israel Binational Science Foundation Grant \#2013408, and Israel Science Foundation Grants \#646/15, \#2140/15, \#2155/15. All computations were conducted on the Hive computer cluster of the Faculty of Natural Science at the University of Haifa.

The funding body had no role in the design of the study, in the collection, the analysis or the interpretation of data.

Availability of data and materials

The sequenced data were deposited in the NCBI SRA data base under the accession PRJNA448217, https://www.ncbi.nlm.nih.gov/bioproject/PRJNA448217.

Authors' contributions

PC carried out all the of the data analyses. EP and PC designed the study. Both authors contributed to writing the manuscript and approved the final manuscript.

Ethics approval and consent to participate

This study complies with the revised Animals Act 1986, as studies on insects require no ethics approval.

No permissions were required to collect the samples in this study.

Consent for publication

Not applicable.

Competing interests

The authors declare that they have no competing interests.

\section{Publisher's Note}

Springer Nature remains neutral with regard to jurisdictional claims in published maps and institutional affiliations.

Received: 29 December 2018 Accepted: 13 May 2019

Published online: 29 May 2019

References

1. Mooney HA, Cleland EE. The evolutionary impact of invasive species. Proc Natl Acad Sci. 2001;98:5446-51. https://doi.org/10.1073/pnas.091093398.

2. Nolte AW, Freyhof J, Stemshorn KCTD. An invasive lineage of sculpins, Cuttos sp. (Pisces, Teleostei) in the Rhine with habitat adaptations has originated from hybridization between old phylogeographic groups. Proc B. 2005:4(September):2379-87.

3. Ellstrand NC, Schierenbeck KA. Hybridization as a stimulus for the evolution of invasiveness in plants? Euphytica. 2006;148:35-46.

4. Ross KG, Trager JC. Systematics and population genetics of fire ants (Solenopsis saevissima complex) from Argentina. Evolution (N Y). 1990:44: 2113-34. https://doi.org/10.2307/2409620.

5. Ross KG, Shoemaker DD. Species delimitation in native south American fire ants. Mol Ecol. 2005;14:3419-38.

6. Shoemaker DD, Ahrens ME, Ross KG. Molecular phylogeny of fire ants of the Solenopsis saevissima species-group based on mtDNA sequences. Mol Phylogenet Evol. 2006;38:200-15.

7. Vander Meer RK, Lofgren CS, Alvarez FM. Biochemical evidence for hybridization in fire ants. Florida Entomol. 1985;68:501-6 http://www.jstor. org/stable/3495147.

8. Vandermeer RK, Lofgren CS. Biochemical and behavioral evidence for hybridization between fire ants, solenopsis-invicta and solenopsis-richteri (hymenoptera, formicidae). J Chem Ecol. 1989;15:1757-65. 
9. Buren WF. Revisionary studies on the taxonomy of the imported fire ants. Journal of Georgia Entomology Society. 1972;7:1-26 http://antbase.org/ants/ publications/6818/6818.pdf.

10. Lofgren CS, Banks WA, Glancey BM. Biology and control of imported fire ants. Annu Rev Entomol. 1975;20:1-30. https://doi.org/10.1146/annurev.en. 20.010175.000245.

11. Caldera EJ, Ross KG, DeHeer CJ, Shoemaker DDW. Putative native source of the invasive fire ant Solenopsis invicta in the USA. Biol Invasions. 2008;10: 1457-79.

12. Morrison LW, Porter SD, Daniels $\mathrm{E}$, Korzukhin MD. Potential global range expansion of the invasive fire ant, Solenopsis invicta. Biol Invasions. 2004;6(2) Callcott 2002:183-91. https://doi.org/10.1023/B:BINV.0000022135.96042.90.

13. Ascunce MS, Yang C-C, Oakey J, Calcaterra L, Wu W-J, Shih C-J, et al. Global invasion history of the fire ant Solenopsis invicta. Science (80- ). 2011;331: 1066-8. https://doi.org/10.1126/science.1198734.

14. Keller $L$, Ross KG. Selfish genes : a green beard in the red fire ant. Nature. 1998;251:573-5.

15. Ross KG, Keller L. Genetic control of social organization in an ant. Proc Natl Acad Sci. 1998;95:14232-7.

16. Wang J, Wurm Y, Nipitwattanaphon M, Riba-Grognuz O, Huang YC, Shoemaker D, et al. A Y-like social chromosome causes alternative colony organization in fire ants. Nature. 2013;493:664-8. https://doi.org/10.1038/ nature11832.

17. Vargo EL, Fletcher DJC. Effect of queen number on the production of sexuals in natural populations of the fire ant, Solenopsis invicta. Physiol Entomol. 1987;12:109-16.

18. Tschinkel WR. The fire ants. Cambridge, Massachusetts: Harvard University Press, Belknap Press; 2006.

19. Ross KG, Shoemaker DD. Estimation of the number of founders of an invasive pest insect population: the fire ant Solenopsis invicta in the USA. Proc R Soc B Biol Sci. 2008;275:2231-40. https://doi.org/10.1098/rspb.2008.0412.

20. Ross KG, Robertson JL. Developmental stability, heterozygosity, and fitness in 2 introduced fire ants (Solenopsis-Invicta and Solenopsis-Richteri) and their hybrid. Heredity (Edinb). 1990;64(July 1987):93-103.

21. Shoemaker DD, Ross KG, Arnold ML. Genetic structure and evolution of a fire ant hybrid zone. Evolution (N Y). 2014;50:1958-76.

22. Pritchard JK, Stephens M, Donnelly P. Inference of population structure using multilocus genotype data. Genetics. 2000;155:945-59.

23. Falush D, Stephens M, Pritchard JK. Inference of population structure using multilocus genotype data: dominant markers and null alleles. Mol Ecol Notes. 2007;7:574-8.

24. Yang Z. Likelihood and Bayes estimation of ancestral population sizes in hominoids usinjg data from multiple loci. Genetics. 2002;162(December): $1811-23$.

25. Zhu T, Yang Z. Maximum likelihood implementation of an isolation-withmigration model with three species for testing speciation with gene flow. Mol Biol Evol. 2012;29:3131-42

26. Dalquen DA, Zhu T, Yang AZ. Maximum likelihood implementation of an isolation-with-migration model for three species. Syst Biol. 2017;66:379-98,

27. Privman E, Cohen P, Cohanim AB, Riba-Grognuz O, Shoemaker D, Keller L. Positive selection on sociobiological traits in invasive fire ants. Mol Ecol. 2018:0-2. https://doi.org/10.1111/mec.14767.

28. Yang S, Wang L, Huang J, Zhang X, Yuan Y, Chen JQ, et al. Parent-progeny sequencing indicates higher mutation rates in heterozygotes. Nature. 2015; 523:463-7.

29. Liu H, Jia Y, Sun X, Tian D, Hurst LD, Yang S. Direct determination of the mutation rate in the bumblebee reveals evidence for weak recombinationassociated mutation and an approximate rate constancy in insects. Mol Biol Evol. 2017:34:119-30

30. Beaumont MA, Zhang W, Balding DJ. Approximate Bayesian computation in population genetics. Genetics. 2002;162:2025-35.

31. Cornuet JM, Santos F, Beaumont MA, Robert CP, Marin JM, Balding DJ, et al. Inferring population history with DIY ABC: a user-friendly approach to approximate Bayesian computation. Bioinformatics. 2008;24:2713-9.

32. Cornuet JM, Pudlo P, Veyssier J, Dehne-Garcia A, Gautier M, Leblois R, et al. DIYABC V2.0: a software to make approximate Bayesian computation inferences about population history using single nucleotide polymorphism, DNA sequence and microsatellite data. Bioinformatics. 2014;30:1187-9.

33. Pyron RA, Costa GC, Patten MA, Burbrink FT. Phylogenetic niche conservatism and the evolutionary basis of ecological speciation. Biol Rev. 2015;90:1248-62.
34. Stouthamer R, Breeuwer JAJ, Hurst GDD. Wolbachia Pipientis: microbial manipulator of arthropod reproduction. Annu Rev Microbiol. 1999;53:71102. https://doi.org/10.1146/annurev.micro.53.1.71.

35. Shoemaker DD, Katju V, Jaenike J. Wolbachia and the evolution of reproductive isolation between Drosophila recens and Drosophila subquinaria. Evolution (N Y). 1999:53:1157-64. https://doi.org/10.2307/2640819.

36. Shoemaker DD, Ross KG, Vargo EL, Werren JH, Keller L. Wolbachia infections in native and introduced populations of fire ants (Solenopsis spp.). Insect Mol Biol. 2000;9:661-73.

37. Sharma KR, Enzmann BL, Schmidt Y, Moore D, Jones GR, Parker J, et al. Cuticular hydrocarbon pheromones for social behavior and their coding in the ant antenna. Cell Rep. 2015;12:1261-71.

38. Evolutionary Genetics of Invertebrate Behavior. 1986; January 1986. https:// doi.org/10.1007/978-1-4899-3487-1.

39. Grillet M, Everaerts C, Houot B, Ritchie MG, Cobb M, Ferveur JF. Incipient speciation in Drosophila melanogaster involves chemical signals. Sci Rep. 2012:2(i):1-11.

40. Yang CY, Kim SJ, Kim J, Kang TJ, Ahn SJ. Sex pheromones and reproductive isolation in five mirid species. PLoS One. 2015;10:1-12.

41. Trajković J, Miličić D, Savić T, Pavković-Lučić S. Sexual selection, sexual isolation and pheromones in Drosophila melanogaster strains after longterm maintaining on different diets. Behav Process. 2017;140(September 2016):81-6.

42. Schultzhaus JN, Bennett CJ, Iftikhar H, Yew JY, Mallett J, Carney GE. High fat diet alters Drosophila melanogaster sexual behavior and traits: decreased attractiveness and changes in pheromone profiles. Sci Rep. 2018;8. https:// doi.org/10.1038/s41598-018-23662-2.

43. Ward PS, Brady SG, Fisher BL, Schultz TR. The evolution of myrmicine ants: phylogeny and biogeography of a hyperdiverse ant clade (Hymenoptera: Formicidae). Syst Entomol. 2015;40:61-81.

44. Ross KG, Krieger MJB, Keller L, Shoemaker DD. Genetic variation and structure in native populations of the fire ant Solenopsis invicta : evolutionary and demographic implications. Biol J Linn Soc. 2007:541-60.

45. Hedrick PW, Parker JD. Evolutionary genetics and genetic variation of haplodiploids and X- linked genes. Annu Rev Ecol Syst. 1997;28:55-83.

46. Zayed A. Effective population size in Hymenoptera with complementary sex determination. Heredity (Edinb). 2004;93:627-30. https://doi.org/10.1038/sj. hdy. 6800588

47. Ross KG, Fletcher JC. Comparative study of genetic and social structure in two forms of the fire ant, Solenopsis invicta (Hymenoptera: Formicidae). Behav Ecol Sociobiol. 1985;17:349-56.

48. Entomol C, Wash C. Revision of the fire ants of the Solenopsis Saevissima. Proc Entomol Soc Washingt. 2018:120:308-411.

49. Baird NA, Etter PD, Atwood TS, Currey MC, Shiver AL, Lewis ZA, et al. Rapid SNP discovery and genetic mapping using sequenced RAD markers. PLoS One. 2008:3:1-7

50. Etter PD, Preston JL, Bassham S, Cresko WA, Johnson EA. Local de novo assembly of rad paired-end contigs using short sequencing reads. PLoS One. 2011;6.

51. Catchen JM, Amores A, Hohenlohe P, Cresko W, Postlethwait JH. Stacks : building and genotyping loci De Novo from short-read sequences. G3\&amp; \#58; Genes|Genomes|Genetics. 2011;1:171-82. https:/doi.org/10.1534/g3.111.000240.

52. Catchen JM. Stacks: an analysis tool set for population genomics. Mol Ecol. 2013;22:3124-40.

53. Langmead B, Salzberg S. Fast gapped-read alignment with Bowtie 2. Nature Methods. 2012;9:357-9.

54. Kopelman NM, Mayzel J, Jakobsson M, Rosenberg NA, Mayrose I. Clumpak: a program for identifying clustering modes and packaging population structure inferences across K. Mol Ecol Resour. 2015;15:1179-91.

55. Evanno G, Regnaut S, Goudet J. Detecting the number of clusters of individuals using the software STRUCTURE: a simulation study. Mol Ecol. 2005;14:2611-20.

56. Keightley PD, Pinharanda A, Ness RW, Simpson F, Dasmahapatra KK, Mallet J, et al. Estimation of the spontaneous mutation rate in Heliconius melpomene. Mol Biol Evol. 2015;32:239-43.

57. Oppold A-M, Pfenninger M. Direct estimation of the spontaneous mutation rate by short-term mutation accumulation lines in Chironomus riparius. Evol Lett. 2017;1:86-92. https://doi.org/10.1002/evl3.8.

58. Aeschbacher S, Beaumont MA, Futschik A. A novel approach for choosing summary statistics in approximate Bayesian computation. Genetics. 2012; 192:1027-47. 
59. Nei M. Estimation of average heterozygosity and genetic distance from a small number of individuals. Houston, Texas: Cent Demogr Popul Genet Univ Texas; 1978. p. 583-90.

60. Weir BS, Cockerham CC. Estimating F-statistics for the analysis of population structure. Evolution (N Y). 1984;38:1358-70.

61. Cornuet J-M, Ravigné V, Estoup A. Inference on population history and model checking using DNA sequence and microsatellite data with the software DIYABC (v1.0). BMC Bioinformatics. 2010;11:401.

62. Shoemaker DD, Ahrens ME, Ross KG, DeHeer CJ, Krieger MJB. Population genetics of the invasive fire ant Solenopsis invicta (Hymenoptera: Formicidae) in the United States. Mol Phylogenet Evol. 2006;38:200-15. https://doi.org/10.1603/0013-8746(2006)99[1213:PGOTIF]2.0.CO;2.

63. Oliver JB, Vander Meer RK, Youssef NN, Samuel A, Pantaleoni E, Mrema FA, et al. Statewide survey of imported fire ant (Hymenoptera: Formicidae) populations in Tennessee'. Tennessee State Univ Sch Agric Consum Sceinces. 2009:149-57.

Ready to submit your research? Choose BMC and benefit from:

- fast, convenient online submission

- thorough peer review by experienced researchers in your field

- rapid publication on acceptance

- support for research data, including large and complex data types

- gold Open Access which fosters wider collaboration and increased citations

- maximum visibility for your research: over $100 \mathrm{M}$ website views per year

At $\mathrm{BMC}$, research is always in progress.

Learn more biomedcentral.com/submissions 